\title{
Hypothesis Testing For Network Data in Functional Neuroimaging (Supplementary Material)
}

\author{
Cedric E. Ginestet*, Jun Li, Prakash Balachandran, \\ Steven Rosenberg, and Eric D. Kolaczyk* \\ Department of Mathematics and Statistics \\ Boston University, Boston, MA.
}

Proof of Theorem 1. Let the matrix $E$ of order $d \times d$ be partitioned in the following manner,

$$
E=\left(\begin{array}{cc}
d-1 & 1 \\
A & v \\
v^{\prime} & x
\end{array}\right) \quad \begin{gathered}
d-1 \\
\end{gathered}
$$

This matrix is assumed to satisfy conditions (1), (2), and (4). We will call the set of such matrices $\mathcal{T}$. Assume that $A$, the top left $(d-1) \times(d-1)$ block of $E$, has nonzero determinant. We want to show that some $d(d-1) / 2$-dimensional ball around $E$ continues to lie in $\mathcal{T}$. Since the rank of $E$ is $d-1$, the last column of $E$ is a linear combination of the first $d-1$ columns. Since the columns of $E$ add to zero and $E$ is symmetric, the rows of $E$ add to zero. For $v^{\prime}=\left(v_{1}, \ldots, v_{d-1}\right)$, we must have

$$
v_{i}=-\sum_{j=1}^{d-1} A_{i j}, \quad \text { and } \quad x=-\sum_{j=1}^{d-1} v_{j} .
$$

Thus, $v$ and $x$ are determined by the entries of $A$.

The matrix, $A$, is symmetric. Thus, it lies in the subspace $S$ of $\mathbb{R}^{(d-1)^{2}}$ of dimension $d(d-1) / 2$ consisting of symmetric matrices. $\operatorname{Det}(A) \neq 0$, so for some matrix $A_{\epsilon}$ in some small neighborhood $U$ of $A$ in $S$, $\operatorname{det}\left(A_{\epsilon}\right) \neq 0$. Each choice of $A_{\epsilon}$ determines a corresponding $v$ and $x$. Conversely, each $E_{\epsilon} \in \mathcal{T}$ sufficiently close to $E$ in the $\mathbb{R}^{d^{2}}$ norm has $\operatorname{det}\left(A_{\epsilon}\right) \neq 0$ and $A_{\epsilon}-A=X$ is symmetric, so $A_{\epsilon}$ and hence $E_{\epsilon}$ is determined by $X$. Thus, a neighborhood of $E$ in $\mathcal{T}$ is bijective to $U$. It is easy to check that this bijection is a diffeomorphism.

${ }^{*}$ This work was supported by a grant from the Air Force Office for Scientific Research (AFOSR), whose grant number is FA9550-12-1-0102. This material is also based upon work supported by, or in part by, the U. S. Army Research Laboratory and the U. S. Army Research Office under contract/grant number W911NF1510440. The data from the 1000 Functional Connectome Project was accessed through the International Neuroimaging Data-sharing Initiative (INDI), which was designed for unrestricted data-sharing via the Neuroimaging Informatics Tool and Resources Clearinghouse (NITRC). We are also indebted to Sean Markan, Lizhen Lin, Emily Stephen and Heather Shappell for useful suggestions and discussion. 
If some other $(d-1) \times(d-1)$ block $B$ of $E$ has nonzero determinant, we note that the top $(d-1) \times(d-1)$ block $A$ of the matrix determines the entire matrix as above. Any small symmetric perturbation $A_{\epsilon}$ of $A$ (with the necessary perturbations of the last row and column to preserve (4)) still satisfies $\operatorname{det}\left(B_{\epsilon}\right) \neq$ 0 . Conversely, any $B \in \mathcal{T}$ sufficiently close to $E$ so that $\operatorname{det}\left(B_{\epsilon}\right) \neq 0$ determines a symmetric perturbation of $A$ as above. Hence, we again obtain a neighborhood of $E$ in $\mathcal{T}$ parametrized by a neighborhood $U$ of $A$ in $S$. This shows that $\mathcal{T}$ is a submanifold of $\mathbb{R}^{d^{2}}$ of dimension $d(d-1) / 2$. The set of matrices satisfying (5) alone is an open convex cone in $\mathbb{R}^{d^{2}}$. When we intersect the submanifold $\mathcal{T}$ with this cone, we get an open submanifold $\mathcal{T}^{\prime}$ of $\mathcal{T}$. Thus $\mathcal{T}^{\prime}$, the set of matrices with (1), (2), (4), (5), is also a submanifold of $\mathbb{R}^{d^{2}}$ of dimension $d(d-1) / 2$.

The space $\mathcal{T}^{\prime}$ has several connected components. A matrix $E_{0}$ with $k$ positive eigenvalues and a matrix $E_{1}$ with $k^{\prime} \neq k$ positive eigenvalues lie in different components, as a path in $\mathcal{T}^{\prime}$ from $E_{0}$ to $E_{1}$ would contain a matrix with a zero eigenspace of multiplicity at least two. Conversely, if $k=k^{\prime}$, then $E_{0}$ and $E_{1}$ are in the same component of $\mathcal{T}^{\prime}$. For the line segment $E_{t}=(1-t) E_{0}+t E_{1}$ stays in $\mathcal{T}^{\prime}$, for every $t \in[0,1]$. Since the components are open, the component of $\mathcal{T}^{\prime}$ satisfying $k=d-1$ is again a submanifold of dimension $d(d-1) / 2$. But this component has condition (3), and so is precisely $\mathcal{L}_{d}$. This proves that $\mathcal{L}_{d}$ is a manifold of dimension $d(d-1) / 2$.

For the convexity statement, conditions $(2)-(5)$ are convex conditions; e.g. for (3), if $A$ and $B$ are positive semidefinite, then

$$
\langle(t A+(1-t) B) v, v\rangle=t\langle A v, v\rangle+(1-t)\langle B v, v\rangle \geq 0
$$

for $t \in[0,1]$ and $v \neq 0$. Clearly, (1) - (5) together is a convex condition. For if $A$ and $B$ satisfy (1) - (5), then $A$ and $B$ come from weighted connected graphs, as does $t A+(1-t) B$. Since a graph is connected iff the rank of the corresponding Laplacian matrix has rank $d-1$, the rank of $t A+(1-t) B$ is $d-1$ for $t \in[0,1]$. Thus $\mathcal{L}_{d}$ is a convex submanifold of $\mathbb{R}^{d^{2}}$.

To show that $\mathcal{L}_{d}$ lies in an affine subset, fix $E \in \mathcal{L}_{d}$. For $k=d(d-1) / 2$, take $k$ distinct points $s_{i}$ in $\mathcal{L}_{d}$, none of them equal to $E$, such that the convex hull of these points contains $E$. (For example, two of the points can be close to $E \pm S$ for a small symmetric matrix $S$.) For generic choices, the $k$ points plus $E$ determine an (affine) $k$-plane $P$, and the convex hull of these points lies in both $P$ and $\mathcal{S}$. Since $P$ and $\mathcal{L}_{d}$ have the same dimension, the open convex hull is exactly a neighborhood of $E$ in $\mathcal{L}_{d}$.

We now show that the plane $P$ is independent of the choice of $E$. Since $\mathcal{L}_{d}$ is convex, it is connected. Take $F \in \mathcal{S}$, let $\ell$ be the Euclidean line segment from $E$ to $F$, and set $E_{t}=(1-t) E+t F \in \mathcal{L}_{d}$. Arguing as above, we find a plane $P_{t}$ containing a neighborhood $V_{t}$ of $E_{t}$ in $\mathcal{L}_{d}$. By compactness, there exist $0=t_{0}, \ldots, t_{n}=1$ with $\cup_{i=0}^{n} V_{t_{i}} \supset \ell$. If $P=P_{0} \neq P_{t_{1}}$, then some line segment from one of the $s_{i}$ 's determining $P_{0}$ to one of the $s_{j}$ 's determining $P_{t_{1}}$ does not lie in $\mathcal{L}_{d}$, a contradiction. Thus $P=P_{t_{1}}$, and by induction, $P=P_{1}$. Since $F$ is arbitrary in $\mathcal{L}_{d}$, it follows that $\mathcal{L}_{d}$ lies in $P$. 
Proof of Corollary 1 . In the notation of the proof of Theorem 1, assume that $E$ has conditions (1), (2), (4), $\left(5^{\prime}\right)$. Then $A$ is symmetric and has $a_{i j} \leq 0$. Thus $A$ is in bijection with the closed "quadrant" $\left\{\left(x^{1}, \ldots, x^{d(d-1) / 2}\right): x^{i} \leq 0\right\}$, which is the basic example of a manifold with corners. If the rank $d-1$ submatrix $B$ of $E$ is not in the top left corner, a relabeling of coordinates moves $B$ to the top left corner. Since the relabeling takes the closed quadrant to a closed quadrant, a neighborhood of $B$ has the structure of a manifold with corners. It is trivial to check that transition maps from chart to chart are smooth. If we impose (3), then as in the previous proof we pick out one connected component of this manifold with corners, and each component is a manifold with corners. The statements on convexity and affine subspaces follow immediately from Theorem 1 , since $\mathcal{L}_{d}^{\prime}$ is a dense subset of $\mathcal{L}_{d}$.

Proof of Theorem 2. Assume the $\ell \times \ell$ block with nonzero determinant occurs in the top left corner; the other cases are handled as in the proof of Theorem 1. Thus let

$$
E=\left(\begin{array}{c|ccc}
\ell & \multicolumn{2}{c}{d-\ell} \\
\hline v_{1}^{\prime} & v_{1} & \ldots & v_{d-\ell} \\
\vdots & b_{1} & \ldots & b_{d-\ell} \\
v_{d-\ell}^{\prime} & & &
\end{array}\right) \quad d-\ell
$$

have conditions $\left(1_{\ell}\right),(2),(4)$. Here, $v_{i}$ is an $\ell \times 1$ column vector, and $b_{i}$ is a $(d-\ell) \times 1$ column vector. The dimension of the set of $\ell \times \ell$ symmetric matrices $A$ with nonzero determinant is $\ell(\ell+1) / 2$. Since the last $d-\ell$ columns must be linear combinations of the first $\ell$ columns, we have

$$
v_{i}=\sum_{j=1}^{\ell} v_{i j} a_{j}, \quad i \in\{1, \ldots, d-\ell\} ;
$$

where $a_{j}$ is the $j^{\text {th }}$ column of $A$. The $v_{i j}$ 's are arbitrary for $i=1, \ldots, d-\ell-1$, but (4) implies that the $v_{d-\ell, j}$ 's are determined by the previous $v_{i j}$ 's. Therefore, we get another $(d-\ell-1) \ell$ degrees of freedom (i.e. dimensions), so the dimension of the space of matrices with $\left(1_{\ell}\right),(2),(4)$ is $\ell(\ell+1) / 2+(d-\ell-1) \ell=d \ell-\ell(\ell+1) / 2$. The argument for adding in conditions (3) and (5) goes as before.

Proof of Theorem 3. The Laplacian CLT considered in this paper is a specialization of a general result due to Bhattacharya and Lin (2016), which considers a metric space $(\mathcal{X}, \rho)$ equipped with a probability measure $Q$. In addition to the conditions stated in the main body of the paper, two further regularity assumptions must be made on the first and second derivatives of the function $\rho^{2}\left(\phi^{-1}(u), x\right)$. These conditions are described below as (A5) and (A6).

Bhattacharya and Lin (2016) have shown that Euclidean coordinates of a Fréchet mean defined on a metric space converges to a normal distribution, under the following assumptions: (A1) the Fréchet mean $\mu$, as described in equation (1) is unique; (A2) $\mu \in A \subseteq \mathcal{X}$, where $A$ is $Q$-measurable, and $\widehat{\mu}_{n} \in A$, almost surely; 
(A3) there exists a homeomorphism $\phi: A \rightarrow U$, for some $s \geq 1$, where $U$ is an open subset of $\mathbb{R}^{s}$; (A4) for every $u \in U$, the map, $u \mapsto h(u ; x):=\rho^{2}\left(\phi^{-1}(u), x\right)$, is twice differentiable on $U$, for every $x \in \mathcal{X}$ outside a $Q$-null set; (A5) for every pair $1 \leq k, l \leq s$, with $u \in U \subseteq \mathbb{R}^{s}$ and $x \in \mathcal{X}$, letting

$$
D_{k} h(u ; x):=\frac{\partial}{\partial u_{k}} h(u ; x), \quad \text { and } \quad D_{k, l} h(u ; x):=\frac{\partial^{2}}{\partial u_{k} \partial u_{l}} h(u ; x),
$$

we require that $\mathbb{E}\left[\left|D_{k} h(u ; x)\right|^{2}\right]<\infty$, and $\mathbb{E}\left[\left|D_{k, l} h(u ; x)\right|\right]<\infty$; moreover, (A6) defining $f_{k, l}(\epsilon, x):=\sup \left\{\left|D_{k, l} h(u ; x)-D_{k, l} h(\phi(\mu) ; x)\right|:|u-\phi(\mu)|<\epsilon\right\}$, we also require modulus continuity, such that $\mathbb{E}\left[\left|f_{k, l}(\epsilon ; Y)\right|\right] \rightarrow 0$, as $\epsilon \rightarrow 0$, for every $1 \leq k, l \leq s$; and finally, (A7) the matrix, $B:=\left\{\mathbb{E}\left[D_{k, l} h(\phi(\mu) ; Y)\right]\right\}_{k, l=1, \ldots, s}$, should be non-singular. Under these conditions, it is then true that the following convergence in distribution holds,

$$
n^{1 / 2}\left(\phi\left(\widehat{\mu}_{n}\right)-\phi(\mu)\right) \longrightarrow N\left(0, B^{-1} V B^{-T}\right),
$$

where $V:=\operatorname{Cov}[D h(\phi(\mu) ; Y)]$ is assumed to be non-singular.

In our setting, we have drawn an iid sample of combinatorial Laplacians from an unknown generating distribution, such that we have $Y_{i} \sim F(\Lambda, \Sigma)$, for every $i=1, \ldots, n$, where $\Lambda$ and $\Sigma$ are the mean Laplacian and the covariance matrix of the upper triangle of $Y$, with respect to some unknown distribution, $F$. Observe that the space of interest is here $\mathcal{L}_{d}^{\prime}$, equipped with the Frobenius distance, as stated in Corollary 1 , thereby forming the metric space, $\left(\mathcal{L}_{d}^{\prime},\|\cdot\|_{F}\right)$. We will see that conditions (A1) - (A4) as well as (A7) are necessarily satisfied in our context. Moreover, we will assume that conditions (A5) and (A6) also hold.

Condition (A1) is readily satisfied, since we have demonstrated that the space of interest, $\mathcal{L}_{d}^{\prime}$, is a convex subspace of $\mathbb{R}^{d^{2}}$; and moreover the arithmetic mean is a convex function on that space by Corollary 1 . Thus, the sample Fréchet mean, $\widehat{L}_{n}$, is unique, for every $n \in \mathbb{N}$. Secondly, we have assumed that the underlying measure gives a non-zero positive probability to a subset $U \in \mathbb{R}^{d^{2}}$, which contains $\Lambda$. Therefore, condition (A2) is satisfied, in the sense, that there exists a subset $A \subseteq \mathbb{M}_{d, d}\left(\mathbb{R}^{+}\right)$, such that $A$ is $\mathbb{P}$-measurable. In addition, since the strong law of large numbers holds for the Fréchet mean (see Ziezold, 1977), we also know that $\widehat{L}_{n} \rightarrow \Lambda$, almost surely; and therefore, $\mathbb{P}\left[\widehat{L}_{n} \in A\right] \rightarrow 1$, as $n \rightarrow \infty$, as required by condition (A2).

For condition (A3), observe that, in our context, the homeomorphism of interest, $\phi: A \mapsto U$, is the half-vectorization function. This takes a matrix

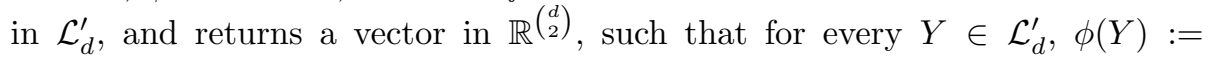
$\operatorname{vech}(Y)$. Specifically, this vectorization is defined by a change of indices, such that for every $i \leq j$, with $1 \leq i, j \leq d$, we have $[\phi(Y)]_{k(i, j)}:=y_{i j}$, with $k(i, j):=(i-1) d+j$. The inverse function, $\phi^{-1}$, is then readily obtained for

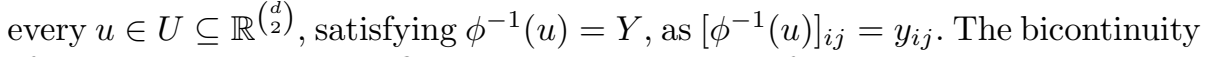
of $\phi$ is hence trivially verified and this map is therefore a homeomorphism.

For condition (A4), the function $h(u ; Y):=\rho^{2}\left(\phi^{-1}(u), Y\right)$, for every $u \in U \subseteq$ 
$\mathbb{R}^{\left(\begin{array}{l}d \\ 2\end{array}\right)}$ and every $Y \in \mathcal{L}_{d}^{\prime}$, outside of a $Q$-null set, is here defined as

$$
h(u ; Y):=\left\|\phi^{-1}(u)-Y\right\|_{F}^{2}=\sum_{i \leq j}^{d}\left(\left[\phi^{-1}(u)\right]_{i j}-y_{i j}\right)^{2},
$$

where the sum is taken over all the pairs of indices $1 \leq i, j \leq d$, satisfying $i \leq j$. The first derivative of this map with respect to the coordinates of the elements of $\mathcal{L}_{d}^{\prime}$ in $\mathbb{R}^{\left(\begin{array}{l}d \\ 2\end{array}\right)}$, is straightforwardly obtained. Setting $X:=\phi^{-1}(u)$, we have

$$
D_{k(i, j)} h(u ; Y):=\frac{\partial}{\partial u_{k(i, j)}}\left\|\phi^{-1}(u)-Y\right\|_{F}^{2}=2\left(x_{i j}-y_{i j}\right) .
$$

The second derivative of $h(u ; Y)$ can be similarly derived for every quadruple, $1 \leq i, j, i^{\prime}, j^{\prime} \leq d$, satisfying $k(i, j) \neq k\left(i^{\prime}, j^{\prime}\right)$. When expressed with respect to $\Lambda \in U$, this gives

$$
D_{k(i, j), k\left(i^{\prime}, j^{\prime}\right)} h(\phi(\Lambda) ; Y)= \begin{cases}2, & \text { if } k(i, j)=k\left(i^{\prime}, j^{\prime}\right), \\ 0, & \text { otherwise. }\end{cases}
$$

It immediately follows that the matrix of second derivatives is $B=2 I$, and hence condition (A4) is verified. In addition, we have assumed that conditions (A5) and (A6) hold in our context. Finally, we have seen that the matrix $B$ is diagonal and hence non-singular, as required by condition (A7).

We can also compute the covariance matrix of the resulting multivariate normal distribution. For this, we require the matrix $V:=\operatorname{Cov}[D h(\phi(\Lambda) ; Y)]$. Given our choice of $\phi$, we need to consider the mean vector of $D h(\phi(\Lambda) ; Y)$, which is given for every $1 \leq i, j \leq n$ by $\mathbb{E}\left[D_{k(i, j)} h(\phi(\Lambda) ; Y)\right]=2\left(\Lambda_{i j}-\mathbb{E}[Y]_{i j}\right)=$ 0 . We can then compute the elements of $V$. For every quadruple $1 \leq i, j, i^{\prime}, j^{\prime} \leq$ $n$, this gives

$$
\begin{aligned}
V_{k(i, j), k\left(i^{\prime}, j^{\prime}\right)} & =\mathbb{E}\left[D_{k(i, j)} h(\phi(\Lambda) ; Y) \cdot D_{k\left(i^{\prime}, j^{\prime}\right)} h(\phi(\Lambda) ; Y)\right] \\
& =4 \mathbb{E}\left[\left(\Lambda_{i j}-Y_{i j}\right)\left(\Lambda_{i^{\prime}, j^{\prime}}-Y_{i^{\prime} j^{\prime}}\right)\right] \\
& =4\left(\mathbb{E}\left[Y_{i j} Y_{i^{\prime} j^{\prime}}\right]-\Lambda_{i j} \Lambda_{i^{\prime}, j^{\prime}}\right)
\end{aligned}
$$

since the cross-term vanishes, after taking the expectation. Therefore, the asymptotic covariance matrix in Theorem 3 is indeed equal to the covariance matrix of the distribution, from which the $Y_{i}$ 's have been sampled. That is, this covariance matrix is given by $B^{-1} V B^{-T}=(2 I)^{-1} V(2 I)^{-1}=\operatorname{Var}[\phi(Y)]=\Sigma$. Therefore, all the conditions of Theorem 2.1 of Bhattacharya and Lin (2016) have been satisfied, and hence $n^{1 / 2}\left(\phi\left(\widehat{L}_{n}\right)-\phi(\Lambda)\right) \rightarrow N(0, \Sigma)$, as stated in Theorem 3.

\section{References}

Bhattacharya, R. and Lin, L. (2016). Omnibus CLTs for Fréchet means and nonparametric inference on non-Euclidean spaces. arXiv preprint arXiv:1306.5806. 
Ziezold, H. (1977). On expected figures and a strong law of large numbers for random elements in quasi-metric spaces. Transactions of the Seventh Prague Conference on Information Theory, Statistical Decision Functions, Random Processes and of the 1974 European Meeting of Statisticians. 